\title{
Ilaria Natali
}

\section{Il medico a teatro: Lucius Junius Brutus di "Mad Nat" Lee}

\begin{abstract}
I: L'originalità di stile e contenuti della produzione di Nathaniel Lee è spesso interpretata principalmente alla luce della presunta malattia mentale dell'autore. Il presente contributo propone un nuovo approccio all'opera di Lee; dimostra come la tragedia Brutus (1680), che contiene una sorta di nosologia delle alterazioni del pensiero o del comportamento umano, sia un testo liminare, sulla soglia tra opera letteraria e trattato medico. L'autore si appropria del discorso scientifico a lui contemporaneo non solo per contestare le più recenti concezioni di patologia mentale, ma anche come strumento per articolare una riflessione sulla condizione del potere politico.
\end{abstract}

Abstract II: The original style and contents of Nathaniel Lee's production have often been interpreted as indicative of the author's alleged mental illness. This contribution proposes a new approach to Lee's works, suggesting that the tragedy Brutus (1680), which contains a sort of nosology of alterations in thought and behaviour, is a liminal text on the border between literature and medical treatise. Lee appropriates the scientific discourse of his time not only to question recent notions of mental disorder, but also to articulate a reflection on the condition of power in the political world.

Keywords: Nathaniel Lee, follia, internamento, Commedia della Restaurazione, storia della medicina.

Nathaniel Lee, madness, internment, Restoration drama, history of medicine.

\section{Follia dell'autore e follia del testo}

Difficile trovare una produzione letteraria che meglio di quella del drammaturgo Nathaniel Lee esemplifichi l'assunto di Carlo Dossi, "tra medicina e letteratura corse sempre amicizia" (1995: 1). Nella forma e nel contenuto, opere quali Nero (1674), Sophonisba (1675), The Massacre of Paris (1679), Caesar Borgia (1679) e Lucius Junius Brutus (1680) rappresentano e rielaborano teorie secentesche del disturbo mentale, con numerosi riferimenti a stati umorali e patologici ${ }^{1}$.

In effetti, non è solo per le copiose descrizioni e performance di follia del suo teatro che Lee attira da secoli lo sguardo della medicina²: sono principalmente la sua figura e la

\footnotetext{
1 Le date indicate fanno riferimento alla prima messa in scena delle opere.

2 Nel presente studio si è scelto di adottare il termine "follia", oramai sorpassato in ambito scientifico, per richiamare un concetto ampio di alterità rispetto alla norma socioculturale. Come madness, anche questo termine ha risonanze in vari ambiti, tra cui "the world of literature and art and of religious belief" (Scull 2015: 14).
} 
sua storia personale ad aver invitato a una lettura dell'opera in chiave interdisciplinare. Della vita di Lee, meglio noto tra i contemporanei come "Mad Nat", si ricorda soprattutto la reclusione nell'ospedale di St. Bethlehem o Bedlam, allora unico istituto pubblico inglese per la cura delle malattie mentali. Tra il 1682 e il 1684, dopo quasi dieci anni a Londra come apprezzato autore teatrale, Lee si trovò quindi costretto a calcare un altro tipo di palcoscenico, il teatro della follia di Bedlam e i suoi spettacoli a pagamento per i visitatori ${ }^{3}$. Ci è giunta indirettamente anche una sua ironica sintesi di come pare si sia svolto il colloquio preliminare all'internamento: "They called me mad and I called them mad, and damn them, they outvoted me" (Porter 2002: 88).

Probabilmente l'autore fu ricoverato perché soggetto a momenti di delirio ed euforia, sintomi che presto i contemporanei collegarono a quell'eccesso di "bombast and extravagant frenzy" (Chambers 1844: 390) che era il veicolo stilistico primario delle sue opere. Per Lee, già definito nel 1675 un "hot-brain'd fustian fool" (Wilmot 1800: 13), l'internamento portò con sé una seconda condanna: la tentazione di stabilire un'interrelazione tra la presunta follia di Lee e le caratteristiche tematico-formali della sua opera fu talmente forte da ridurre l'intera reputazione dello scrittore ad una cronaca della sua malattia. Quando l'opera di Lee non era considerata un sintomo di pazzia, si suggeriva che la sua modalità espressiva 'da folle' gli aveva aperto la strada verso Bedlam: “Nathaniel Lee”, dice Anthony Wood nel 1684, "endeavoring to reach high in expression in his plays, broke his head and fell distracted" (Wilson 1968: 89).

La condanna 'estetica' è quella che si rivela più drammatica e persistente per Lee e la sua fortuna letteraria. A quasi due secoli dalla morte dell'autore, le sue opere continuavano a essere viste come manifestazione di "an impetuosity in which it is easy to discover the traces of incipient insanity" (Ward 1875: 543). Anche in tempi più recenti, la critica non ha abbandonato il proposito di rilevare il carattere farneticante dei suoi plays in rapporto ai principali modelli espressivi dell'epoca, i più misurati di Dryden e Otway ${ }^{4}$. Accanto ad apporti utili e innovativi, sullo studio dell'opera di Lee si sono riversati nel tempo approcci che sembrano esemplificare quelli che oggi consideriamo i principali rischi interpretativi dell'indagine interdisciplinare tra letteratura e (storia della) medicina. Lee è stato oggetto, infatti, di tentativi anacronistici di 'diagnosi a distanza', spesso condotti secondo concetti di salute mentale poco consoni al contesto pre-psichiatrico del mondo da lui conosciuto e descritto.

D'altra parte, la produzione di Lee ha anche sollecitato fruttuosi percorsi di ricerca multidirezionali, spesso volti ad affrontare l'annoso problema della sua collocazione in rapporto alle convenzioni più diffuse nel teatro della Restaurazione. Dal momento che i tentativi di far rientrare le tragedie entro i canoni aristotelici hanno evidenziato alcune forzature, si è pensato che Lee scriva in limine tra tragedia e commedia, idea che giustificherebbe l'impiego frequente di toni dissacranti e satirici ${ }^{5}$. Tuttavia, nei drammi di Lee si può rico-

\footnotetext{
3 Per maggiori informazioni sulle visite a pagamento e il mad show di Bedlam nel Sei-Settecento, cfr. ad es. Porter 2000 e Andrews et al. 1997.

4 Cfr. ad es. Brown (1983: 385), Frank (2003: 109) e Danby (2014: 191-208).

5 Cfr. Verdurmen (1989: 83-84) e Brown (1983: 385-401).
} 
noscere anche "a [...] destruction of the clichés of early Restoration tragicomedy" (Hughes 2008: 208); non è senza riserve, inoltre, che possono essere annoverati tra gli "horror plays" discussi da Hermanson (2014: 14). In definitiva, nessuna categorizzazione sembra funzionare appieno, e nessuna riesce a dar conto in modo soddisfacente dell'originalità di temi, immagini e modi espressivi impiegati da Lee. L'eccentricità dei personaggi e l'alterità dello stile delle sue opere in rapporto ai canoni tardo-secenteschi hanno finito, quindi, per essere giustificate supponendo che le stesse qualità caratterizzassero il pensiero dell'autore.

A prima vista, non pare che mettere in scena la follia a fine Seicento costituisca uno scarto dalla norma. Il teatro della Restaurazione, così come quello dei decenni precedenti, è invaso da regnanti che perdono il senno, innamorati malinconici, pazzie simulate. È chiaro che Lee accoglie e rielabora la tradizione recente, inclusa quella shakespeariana, ma con alcuni accorgimenti: lo stato di disordine mentale è raramente innescato da una frattura nell'ordine costituito o un'azione distruttiva, e si fa piuttosto primo motore della tragicità delle vicende, come avviene in Brutus e The Massacre of Paris. Ancor più originale, a mio avviso, è il livello di pervasività della rappresentazione della patologia mentale nell'opera di Lee: l'eccentricità è sia tecnica di composizione letteraria, sia tratto distintivo di più di un personaggio in ciascun play. Per Lee, il folle è nel mondo e lo domina, anche in senso letterale, poiché le anomalie comportamentali caratterizzano non solo chi partecipa alla società, ma soprattutto chi vi ricopre incarichi di potere, come evidente in Nero o Caesar Borgia. La pervasività dello stato patologico è anche data dall'insistenza sui dettagli con cui ciascuna condizione è rappresentata: lo stato fisico-mentale dei personaggi è in genere illustrato con dovizia sintomatologica e specifici riferimenti al discorso medico-scientifico dell'epoca.

È opportuno ricordare, a questo punto, che nell'Inghilterra del tardo Seicento coesistevano e si intrecciavano diverse nozioni di disturbo mentale, che non mi soffermo qui ad illustrare per ragioni di spazio e per averne già trattato altrove ${ }^{6}$. Il discorso medico accostava idee antiche e recenti, ivi incluse teoria degli umori, circolazione del sangue, spiriti animali o vitali, fumi e vapori. La concezione di alterazione mentale, temporanea o permanente, era una struttura dinamica all'interno della quale non vigeva un principio di esclusione che scartasse i punti di vista poco in armonia o persino incompatibili tra loro ${ }^{7}$; di conseguenza, anche Lee ricorre a vari sistemi di riferimento contemporaneamente, derivando idee, per esempio, da Robert Burton, William Harvey e Thomas Willis. A suscitare interesse, tuttavia, non sono tanto le fonti specifiche da cui Lee trae materiale per le proprie rappresentazioni, ma il modo stesso in cui è articolato il suo discorso interdisciplinare e come si interseca con la tradizione letteraria e culturale.

Non è da escludere che l'interesse di Lee per l'ambito medico derivi da una sua consapevolezza di essere percepito in termini di alterità o eccentricità mentale rispetto alla norma socioculturale del proprio tempo già prima del ricovero a Bedlam. Credo, tuttavia, che qualsiasi valutazione, presente o passata, sulla condizione mentale di un autore sia poco rilevante

\footnotetext{
6 Cfr. Natali 2016.

7 Rileva Michael MacDonald, "Physicians tried to maintain their scientific respectability by accepting some modern ideas and marrying them to ancient remedies, a match that was more attractive politically than philosophically" (1981: 197).
} 
ai fini dell'analisi della sua opera: in linea con Allan Ingram, infatti, ritengo che la follia trovi espressione in letteratura sempre e solo in quanto rappresentazione (Ingram \& Faubert 2003: 4). L'approccio più produttivo, nota Shoshana Felman, è esplorare le "sophisticated ways by which literature communicates with madness" (2003: 4); è per presentare alcune ipotesi a tale riguardo che di seguito vorrei concentrare l'attenzione su una delle opere più note di Lee, Lucius Junius Brutus, messa in scena nel 1680 e pubblicata l'anno successivo.

\section{Rappresentazioni di follia in Brutus}

Poco dopo la sua prima rappresentazione sotto il regno di Carlo II, Brutus fu censurato per "Scandalous Expressions \& Reflections vpon Ye Government" (TNA LC 5/144) ${ }^{8}$. I motivi della censura sembrano abbastanza evidenti: parlare della fondazione della Repubblica romana ripetendo la parola "Commonwealth" per tredici volte difficilmente non avrebbe suscitato allarme a soli venti anni dalla Restaurazione, specie dopo il presunto complotto papista del 1678. È forse a seguito della censura che si è spesso pensato a Brutus come a un testo di propaganda pro-repubblicana, focalizzando l'attenzione sul presunto orientamento

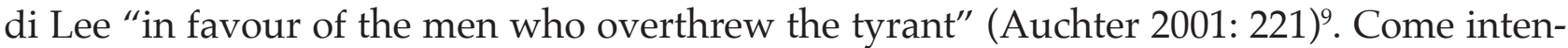
do dimostrare di seguito, tuttavia, quest'opera non rappresenta un'ennesima favilla della polemica repubblicana ormai quasi spenta. Per poter essere considerata propagandistica, la tragedia dovrebbe soddisfare almeno una condizione essenziale; proporre un'immagine di Bruto positiva, se non esemplare; tale condizione, a mio parere, non è soddisfatta nel testo.

Il contesto del play è abbastanza noto. Secondo Ab Urbe condita libri di Tito Livio ${ }^{10}$, una delle fonti principali di Lee, Lucio Giunio si finge pazzo per proteggersi dalla tirannia di Tarquinio il Superbo, guadagnandosi così l'appellativo di "Brutus" ("irrazionale", "stolto"). Appreso dello stupro di Lucrezia e rivelata la propria dissimulazione, è a capo della rivolta che schiaccerà l'ultimo re. Nella storia si potrebbero già configurare i tratti di un tipico revenge play, se non fosse che, nella versione di Lee, le vicende di Lucrece non sembrano essere determinanti per spronare Brutus all'azione (Lee 1967: I.i.94-111, 12). In modo piuttosto inconsueto per il teatro secentesco, inoltre, Brutus rivela l'inganno della sua condizione a sole dodici brevi battute dalla propria comparsa in scena $(\mathrm{I} .1 .198-201,16)^{11}$. È principalmente da quanto dice al figlio Titus che apprendiamo come sinora abbia interpretato "all variety of aping madness" (I.i.121, 13), apparentemente simulando non tanto una vera e propria istanza di follia, quanto la patologia che nel Seicento si definiva "idiocy" e costituiva un impedimento generico delle capacità intellettive ${ }^{12}$. La tempestiva rivelazione di Brutus permette al drammaturgo di porre l'accento sul momento dell'azione e del cambiamento, anziché concentrarsi sulla

\footnotetext{
8 Il testo del manoscritto è citato, ad es., in Nicoll 1952: 10n.

9 Per un'interpretazione del testo come propaganda repubblicana o whig, cfr. anche Loftis 1967; secondo Hayne, Brutus rappresenta i rischi dell'estremismo repubblicano (1996).

10 Cfr. Livio 1841: I.lvi-lx, 133-142.

11 Nel conteggio delle battute, non si tiene conto del soliloquio in I.i.94-133, in cui non c'è finzione di follia.

12 Secondo Essay Concerning Human Understanding di Locke, "mad Men put wrong Ideas together, and so make wrong Propositions, but argue and reason right from them: But Idiots make very few or no Propositions, and reason scarce at all"' (1975: XI.ii.13, 161). Sull'argomento cfr. anche Andrews (1988: 66).
} 
corruzione del regno di Tarquin. Soprattutto, a Lee dovette sembrare superfluo soffermarsi sullo spettacolo della simulazione di Brutus quando molto più spunto offre il fatto che tale simulazione può nascondere una reale patologia o persino esserne un sintomo.

Davanti al figlio, Brutus subisce una metamorfosi degna del testo ovidiano, in cui muta non solo atteggiamento ma persino aspetto: Titus osserva che "no part of him" rimane "The same; nor eyes, nor mien, nor voice, nor gesture!" (I.i.224-5, 17). La trasformazione, tuttavia, non porta ad un'immagine 'normalizzata' di Bruto, ma segna piuttosto il passaggio da una rappresentazione di alterità ad un'altra:

[...] No, degenerate boy,

Brutus is not the same; the gods have waked him

From dead stupidity to be a scourge,

A living torment to thy disobedience.

Look on my face, view my eyes flame, and tell me

If aught thou seest but glory and revenge,

A blood-shot anger, and a burst of fury,

When I but think of Tarquin [...] (I.i.228-235, 17).

Occhi in fiamme, il sangue che ribolle, Brutus incarna il cliché del raging fool secentesco, caratterizzato da rabbia furente, esaltazione e violenza. I segnali della sua manic insanity sono, poi, ripresi e rafforzati nel corso dell'intera tragedia anche attraverso le percezioni altrui; ad esempio, Titus nota che "the gods light'ned from his awful eyes, / and thundered from his tongue" (II.i.488-9, 41) e Sempronia si interroga: "is he not mad?", riferendosi alla sete di sangue che ora "rains from his own bowels" (V.i.92-93, 84). Effettivamente, Brutus ha raggiunto un nuovo stato, anch'esso suggerito e definito dal proprio nome: la brutalità incontrollabile che infiamma il folle $\mathrm{e}^{13}$. La forza del tradizionale eroe erculeo si fonde qui con la frenetica ed iraconda sregolatezza della pazzia, dando vita ad un nuovo ibrido, una sorta di herculean madness.

Come si evince sin dalla Summa theologiae di Tommaso D'Aquino (1969, III, q.15, a.9, 70), la rabbia, se giustificata, non è segno di alterazione morale o mentale, e può essere anzi tratto del virtuoso; lo ricorda anche Tiberius nel testo di Lee, "You may be angry, and may be forgiven" (II.i.12, 26). Ma sono continui i momenti del dramma in cui la virtù e le ragioni di Bruto sono messe esplicitamente in discussione, persino da lui stesso:

O Rome, O mother, be thou th'impartial judge

If this be virtue, which yet wants a name [...] (I.i.130-1, 13).

La furia di Brutus non può configurarsi come virtù perché Lee non le attribuisce un motivo chiaro e razionale. Il protagonista, suggerisce anche David Vieth, ci appare incoerente, poiché il suo odio intenso verso Tarquin si scatena d'improvviso, dopo ben venti anni

13 L'immagine della follia di Brutus è derivata essenzialmente da Burton (1638: 104-105); idee molto simili compaiono in Willis (1685: 479). 
di silenzio e dissimulazione, senza chiaro innesco (1975: 62-63). Altrettanto oscura rimane la ragione che spinge Brutus ad impedire a Titus di unirsi alla novella sposa Teraminta, figlia illegittima di Tarquin, donna che, per ammissione dello stesso Brutus, è "chastely good" e "Without the smallest tincture of her father" (II.i.321-2, 36). Naturalmente, l'atto più terribile e ingiustificabile dell'eroe è l'uccisione dei propri figli, che insiste siano condannati ad una morte senza onore. È legittimo dubitare, quindi, che Lee stia portando avanti una linea politica antimonarchica: più che all'opera di un salvatore della patria, sembra di assistere all'imperversare di un nuovo tiranno (così lo definiscono spesso i figli e Teraminta, es. II.i.363, 37 e V.i.116, 85) spinto dalla s-ragione.

La follia di Brutus è solo un tassello in un complesso mosaico di riferimenti medici e biologici che domina il play. Lee costruisce un'anatomia della mente e del corpo umano: l'immaginario del dramma, nota Vieth, è "viscerale" (1975: 64), pervaso da idee di smembramento e sventramento. Sono menzionati, inoltre, i "fumes" della cattiva digestione (IV.i.163, 66), disequilibri degli umori, degli spiriti vitali (es. I.i.101, 12; 173, 15; 440, 24; II.i.362, 37; IV.i.513, 78) e del flusso sanguigno (es. I.i.102, 12 e II.i.439, 40) ${ }^{14}$. Se "multitude is a mad thing" (II.i.31, 27), come sostiene Fabritius, è perché ciascun individuo che costituisce la folla presenta, in modo più o meno temporaneo, una mancanza di lucidità. Per esempio, la gens Tarquinia e la corte partecipano di una comune forma di squilibrio: proni ad eccessi bestiali e brutali, sono guidati da istinti primari e dominati da impulsi erotici. Lucrece, violata da Sextus, di lui ricorda i "red and sparkling eyes, / The flushing blood that mounted in his face, / The trembling eagerness that quite devoured him" (I.i.367-9, 22). Questo tipo di follia distruttrice non si riscontra nei testi medici secenteschi, ma richiama senz'altro la concezione di "alienatione" animalesca che Marsilio Ficino attribuisce all'eccesso di "sangue adusto" in El libro dell'amore (VII.iii, 188).

Con l'occhio di un medico piuttosto che di un drammaturgo, già in apertura di Brutus Lee presenta una coppia di innamorati che si definisce in termini di alterità dalla norma comportamentale. Queste sono, infatti, le parole rivolte da Titus a Teraminta:

O Teraminta, why this face of tears?

Since first I saw thee, till this happy day,

Thus hast thou past thy melancholy hours,

Even in the court retired; stretched on a bed

In some dark room, with all the curtains drawn;

Or in some garden o'er a flow'ry bank

Melting thy sorrows in the murmuring stream [...] (I.i.1-7, 9).

Teraminta mostra i sintomi della malinconia flegmatica illustrata da Burton ${ }^{15}$, mentre

\footnotetext{
14 Lee sembra trarre nozioni da più fonti, ma la principale è ancora Anatomy of Melancholy, che illustra i "Fumes arising from the stomack" (1638: 198), parla di disequilibrio negli spiriti vitali e nel sangue (es. 1638: $15,34)$.

15 Scrive Burton: "For example, if [melancholy proceeds] from fleagme [...] it stirres up dull Symptomes, and a kinde of stupiditie, or impassionate hurt: they are sleepy, saith Savanarola, dull, slow, cold, blockish, [...], they are much given to weeping and delight in waters, ponds, pooles, rivers [...]" (1638: 191).
} 
Titus attraversa due diverse condizioni nel play, incarnando le principali tipologie di "madness" individuate da Hobbes (2011: 86-7). Dapprima lo vediamo, come dice il padre, vittima di "the heated channels of [his] veins" (Lee 1967: II.i.351, 37), vale a dire in preda a una smisurata "ecstasy" ed "extravagant passion" (I.i.34-5, 10) per Teraminta: con il proprio febbrile ardore si propone persino di riscaldare l'amata, dominata dai freddi umori della malinconia ("Cold as thou art", I.i.38, 10). Dopo che Brutus mette in evidenza le inopportune "raptures" e gli eccessi patologici di Titus (I.i.198-99, 16), impedendogli di consumare il matrimonio appena celebrato, quest'ultimo piange e trema "drowned in his melancholy" (III.iii.14, 55), affezione che lo accompagnerà fino a una morte cercata e desiderata. La follia di Titus è accecante e autodistruttiva, ma non al punto di impedirgli di riconoscere, a sua volta, l'irragionevolezza del padre, e biasimarlo perché intende ucciderlo in maniera così umiliante (IV.i.534-547, 78). È particolarmente innovativo che, anche se i singoli stati patologici di Titus rimangono isolati e autonomi, alla sua alterità mentale sia attribuito uno status di processo e una capacità di dinamismo raramente concettualizzata dalla scienza dell'epoca.

Attraverso i personaggi di Brutus, in definitiva, Lee sviluppa una nosologia del disturbo mentale o comportamentale che poggia su modelli preesistenti per arricchirli di nuove declinazioni e rielaborarli in combinazioni originali. Si fa strada l'ipotesi che il testo non attraversi solo diversi modelli e convenzioni letterarie del proprio tempo, ma soprattutto che si situi in una posizione liminale tra opera teatrale e trattato medico. Nella tragedia, immaginario letterario e scientifico si fondono fino a farsi indistinguibili, in una modalità ibrida che pare suggerire una natura fittizia comune alla creazione artistica e al discorso della medicina. Se la figura semileggendaria dell'eroe Brutus può sembrare patologica agli occhi del Seicento è perché nell'identificare i sintomi della follia si attua una procedura arbitraria e basata su criteri variabili, dipendenti dal contesto epocale. Non senza intento satirico, quindi, Lee ricostruisce una realtà distante nel tempo e nello spazio reinterpretando i comportamenti dei personaggi alla luce di nozioni moderne di salute e malattia; così il virtuoso mondo dell'antica Roma si trasforma in una sorta di Narrenschiff o, in termini più moderni, di Bedlam. È rispetto a quest'ultimo scenario che spicca una differenza fondamentale: i folli di Lee non sono internati o isolati, non sono in cerca di cure e terapie. L'alterazione del pensiero o del comportamento è, piuttosto, accettata criticamente e consapevolmente come inevitabile nel palcoscenico del mondo.

\section{Il folle, il medico, il sovrano}

Brutus mette in discussione la stabilità del confine tra normalità e anormalità di pensiero e comportamento appropriandosi dello stesso discorso che si occupa di tracciare e descrivere tale confine. Lee sembra percepire il concetto di patologia come profondamente radicato in uno specifico quadro sociale e culturale; dal momento che il disturbo mentale ha senso solo se contestualizzato o storicizzato, sottolinea la transitorietà dei suoi modelli, mettendo in discussione la legittimità di qualsiasi lettura della follia.

Queste idee sono di grande attualità nel momento in cui Brutus le affronta. Sebbene a livello giuridico varie forme di difficoltà fisica, mentale o sociale risultino ancora regolamentate in modo indifferenziato dal Poor Law Act del 1601, nella seconda metà del Sei- 
cento si vanno affermando nuove linee di condotta riguardanti la specifica gestione e cura del disagio mentale. Sempre più spesso si sente la necessità di ricorrere all'internamento e all'allontanamento dalla società del diverso, come dimostrano i progressivi ampliamenti cui è sottoposto l'ospedale di Bedlam: l'espansione iniziata negli anni Trenta del Seicento culmina nella totale ricostruzione del 1676, quando gli spazi per i pazienti si moltiplicano in un nuovo edificio maestoso e regale.

Mentre la società si prepara a isolare l'alterità mentale, il mondo scientifico elabora nuove categorizzazioni e soprattutto nuove terapie fisiche estremamente invasive finalizzate a ricondurre il paziente alla 'ragione'. Particolarmente rappresentative di questa tendenza sono le indicazioni offerte da Thomas Willis in De Anima Brutorum (1672): si apprende dalla versione inglese del trattato che qualsiasi cura deve essere accompagnata da isolamento, e compito del medico è usare forza pari e contraria a quella dimostrata dal paziente, così che "to desperate Love ought to be applied or shrewed indignation and hatred" (1683: 193). Inoltre, "to correct and allay the furies and exorbitancies of the Animal Spirits" il medico necessita di "threatnings, bonds, or strokes, as well as Physick" (1683: 206). Che la linea di confine tra cura e sopruso si stesse facendo molto sottile era già emerso agli occhi dell'opinione pubblica nel 1632, quando l'unico medico responsabile di Bedlam, Helkiah Crooke, fu al centro di uno scandalo per trattamenti barbarici e palesi abusi nei confronti dei ricoverati.

Lee percepisce il sintomo di un importante cambiamento nella concezione e nella gestione del disturbo mentale in Inghilterra, e risponde a questa situazione elaborando in Brutus una riflessione sui concetti di malattia e di potere. Tale riflessione finisce, poi, per ampliarsi a trattare un problema storico più vasto e complesso, poiché la tragedia stabilisce una rete di analogie tra la pratica della medicina e quella della ragion di stato. Il discorso medico si fa, quindi, pretesto e strumento per articolare anche un dibattito politico.

In Brutus, il governo è un organismo la cui salute dipende da ciascuno dei suoi componenti. A rendere palese la corrispondenza tra patologia umana e i problemi 'organici' di Roma è Titus, che spiega a Teraminta come il padre si sia autoproclamato rimedio per la follia dominante:

The body of the world is out of frame, The vast distorted limbs are on the rack And all the cable sinews stretched to bursting, The blood ferments, and the majestic spirit, Like Hercules in the envenomed shirt, Lies in a fever on the horrid pile.

My father, like an Aesculapius

Sent by the gods, comes boldly to the cure.

But how, my love? By violent remedies,

And says that Rome, ere yet she can be well, Must purge and cast, purge all th'infected humors

Through the whole mass; and vastly, vastly bleed (II.i.436-47, 40). 
Brutus, come Thomas Willis e altri medici di fine Seicento, propone una cura di "violent remedies" che non sembra promettere successo, ma solo sofferenza. Non va dimenticato, inoltre, che il novello Asclepio è tanto disfunzionale quanto il mondo che ironicamente si ripromette di sanare: in Brutus, quindi, prende corpo la tradizionale figura del quack, un medico ciarlatano e folle simile a quelli che popolano la Narrenliteratur. Da una prospettiva politica, il sanatore della patria non è meno tirannico e brutale del sovrano che intende spodestare, e giustifica l'uso della violenza con la ragion di stato, pur senza invocarla apertamente. Lee suggerisce che questa modalità di pensiero, nel mondo medico così come in quello politico, traversa una soglia sensibile, in cui si annidano storture e rischi di sopruso: si vanno ad intaccare valori di giustizia e libertà, e quella fondamentale legge di natura che secondo Locke è "the preservation of all mankind" (2004: 5).

La metafora anatomica, già ampiamente sfruttata almeno sin dalla Repubblica di Platone, non ha molto di innovativo; tuttavia, anche in questo caso, Lee declina elementi della tradizione nell'attualità politica e sociale del proprio tempo. Alla monarchia restaurata sembrano alludere le parole di Vinditius, secondo il quale "the people in the body politic are but the guts of government" senza "head" (I.i.294-297, 19): come nota J. M. Armistead, "when Charles I was beheaded, the state itself became headless" (1979: 26) ed entrò in crisi il concetto che il re potesse rappresentare la mente dell'organismo politico. Soprattutto, Carlo II, re e medico del corpo malato dell'Inghilterra, era considerato un guaritore anche fuor di metafora, poiché dopo l'Interregno aveva reintrodotto la cerimonia del tocco regale, che si credeva sanasse miracolosamente una serie di malattie. Attraverso questa rete di riferimenti, Brutus mette in discussione la fiction di onnipotenza semidivina della regalità inglese: l'immagine del sovrano si rivela improvvisamente nella sua superba fallibilità umana ${ }^{16}$.

Carlo II è indubbiamente uno degli obiettivi di Lee in quella che si configura sempre più come una satira della sovranità, ma non è il suo unico obiettivo. Il discorso politico in Brutus ha respiro ampio, stimola una riflessione generale sulle cariche e le funzioni di governo, su tutta la condizione del potere piuttosto che su figure specifiche che lo rappresentano. A suggerire questa ampiezza di vedute sono le relazioni intertestuali che Lee stabilisce tra la propria opera e gli scritti di Niccolò Machiavelli, indicati come fonte di ispirazione per Brutus nella dedica iniziale al Conte di Dorset (Lee 1967: 4, 45-47). Nei Discorsi, infatti, Machiavelli loda la "simulazione della stultizia" di Bruto, portandolo ad esempio a quanti intendano ribellarsi ad un principe: la finzione consente di "prima misurare e prima pesare le forze loro" per poi passare all'attacco (III.2, 2000: 229, 265). Il Brutus di Lee si discosta da questo modello di comportamento: non raccoglie le proprie forze prima di svelare l'inganno della finta follia, non attende di aver organizzato razionalmente la rivolta, pare solo spinto da un furor irresistibile. Che la ragione machiavellica sia in lui assente è ulteriore motivo di ironia, e sottolinea l'instabilità e inefficacia del suo comportamento.

Con l'immagine del mondo come corpo malato, Lee amplia e rinsalda la connessione con l'opera di Machiavelli, che spesso nel Principe e nei Discorsi ascrive poteri di guarigione

16 La metafora Stato/organismo richiama anche Microcosmographia di Crooke, lo stesso medico di Bedlam al centro dello scandalo del 1632, secondo il quale il corpo umano è "rule and square of all bodies" e l'anatomia apre la strada a tutto lo scibile umano (1615: 12). 
del corpo politico a chi lo governa. Per Machiavelli, la "medicina" deve essere sempre assunta "a tempo" dallo Stato (III.26, 1962: 20) e perché il malanno non "ammazz[i ... il] corpo" (III.1, 2000: 224), spesso è necessario ricorrere al "ferro" (I.58, 2000: 127), vale a dire alle esecuzioni. Il politico-medico, come Brutus o Thomas Willis (che ho eletto qui a rappresentante dell'alienista secentesco), deve essere pronto a colpire, tagliare e agire con decisione a seconda di quanto richiede la gravità della patologia. Ė questo cinismo privo di basi morali che Lee sembra mettere in discussione tanto nell'ambito della medicina quanto in quello della politica: in caso di necessità il potere si può fare eccessivo e illegittimo, ogni arbitrio sembra possibile, ogni violenza giustificata e ogni sopruso tollerabile.

Leggendo Brutus alla luce delle relazioni con l'opera di Machiavelli risulta chiaro come la riflessione politica di Lee vada oltre le specificità del proprio tempo per mettere in evidenza che, in qualsiasi tipo di governo, chi ha autorità decisionale può godere di eccessivi poteri e scarse competenze. Per citare Bacon, "as there are mountebanks for the natural body, so there are mountebanks for the politic body; men that undertake great cures, and perhaps have been lucky in two or three experiments, but want the grounds of science, and therefore cannot hold out" (1972: 35). La tragedia di Lee destabilizza il paradigma della sovranità nel capo di Stato e della sua emanazione, il medico moderno, che acquisisce potere assoluto sul suo paziente; a queste figure è lecito esercitare un pragmatismo bestiale come a Brutus, che non è spinto da pietas ma da violenza, furia, barbarie e ambizione.

Rimangono aperte, nel testo di Lee, una serie di domande. Per esempio, chi protegge l'individuo dall'arbitrio di quanti detengono il potere, o dalla fallibilità umana, compresa quella del sovrano e del medico? Brutus non propone risposte, la sua conclusione è ambigua e non allude ad alcun recupero di un equilibrio. Un tiranno è sconfitto, ma un altro si appresta a prendere le redini del mondo, negando qualsiasi senso di speranza in un miglioramento o una rinascita. Nessuno, in Brutus, sembra aver imparato dai propri errori, che si suppone continueranno a ripresentarsi ciclicamente. Rimane solo l'amaro sorriso dello spettatore davanti all'ironica promessa dell'iracondo e ambizioso Brutus di costruire un futuro "where rancor and ambition are extinguished" (III.ii.62, 51).

\section{BIBLIOGRAFIA}

Andrews, Jonathan et al. 1997. The History of Bethlem. London-New York: Routledge. Andrews, Jonathan. 1998. Begging the Question of Idiocy: The Definition and Socio-cultural Meaning of Idiocy in Early Modern Britain: Part 1. History of Psychiatry, 9: 65-95.

Armistead, J. M. 1979. Nathaniel Lee. Boston: Twayne.

Auchter, Dorothy. 2001. Dictionary of Literary and Dramatic Censorship in Tudor and Stuart England. Westport (CT): Greenwood Press.

Bacon, Francis. 1972 [1597]. Of Boldness. Michael J. Hawkins ed. Essays. London: Dent. Brown, Richard. 1983. Heroics Satirized by “Mad Nat. Lee”. Papers on Language and Literature, 19, 4: 385-401.

Burton, Robert (pseud. Democritus Junior). 1638 [1621]. The Anatomy of Melancholy: What it is, with all the kinds causes, symptomes, prognostickes $\mathcal{E}$ seuerall cures of it. Oxford: Henry Cripps. 
Chambers, Robert \& William Chambers eds. 1844. Cyclopædia of English Literature (Vol. 1). Edinburgh: William \& Robert Chambers.

Crooke, Helkiah. 1615. Microcosmographia: A Description of the Body of Man. London: Jaggard. D'Aquino, Tommaso. 1969. La somma teologica. Traduzione e commento a cura dei domenicani italiani; testo latino dell'edizione leonina. Vol. XXIV: Incarnazione: b) difetti assunti e implicanze. Firenze: Salani.

Danby, Jennifer Renee. 2014. Burning and Stoic Men: Mad Rants and the Performance of Passionate Pain in the Plays of Nathaniel Lee, 1674 to 1678. Kathryn Lowerre ed. The Lively Arts of the London Stage, 1675-1725. Farnham: Ashgate, 191-208.

Dossi, Carlo. 1995 [1883]. Dal calamajo di un mèdico. Isella Dante (a cura di). Opere. Milano: Adelphi.

Felman, Shoshana. 2003 [1985]. Writing and Madness (Literature/Philosophy/Psychoanalysis). Palo Alto: Stanford University Press.

Ficino, Marsilio. 1987 [1491]. El libro dell'amore. Sandra Niccoli (a cura di). Firenze: Olschki. Frank, Marcie. 2003. Gender, Theatre, and the Origins of Criticism: From Dryden to Manley. Cambridge: Cambridge University Press.

Hayne, Victoria. 1996. "All Language Then is Vile": The Theatrical Critique of Political Rhetoric in Nathaniel Lee's Lucius Junius Brutus. ELH, 63, 2: 337-365.

Hermanson, Anne. 2014. The Horror Plays of the English Restoration. Farnham: Ashgate.

Hobbes, Thomas. 2011 [1651]. Leviathan. A. P. Martinich \& Brian Battiste eds. New York: Broadview.

Hughes, Derek. 2008 [2001]. Heroic Drama and Tragicomedy. Susan J. Owen ed. A Companion to Restoration Drama. Oxford: Blackwell, 195-210.

Ingram, Allan \& Michelle Faubert. 2003. Cultural Constructions of Madness. New York: Palgrave.

Lee, Nathaniel. 1967 [1680]. Lucius Junius Brutus. John Loftis ed. Lincoln: University of Nebraska Press.

Livio, Tito. 1841. Titi Livii Patavini historiarum ab Urbe condita libri / La storia romana di Tito Livio. Luigi Mabil (a cura di) (Vol. 1). Venezia: Giuseppe Antonelli.

Locke, John. 1975 [1690]. Essay Concerning Human Understanding. P. H. Nidditch ed. Oxford: Claredon.

Locke, John. 2004 [1690]. The Second Treatise of Government. Jospeh Carrig ed. New York: Barnes \& Noble.

Loftis, John. ed. 1967. Introduction. Nathaniel Lee, Lucius Junius Brutus. Lincoln: University of Nebraska Press, xi-xxiv.

MacDonald, Michael. 1981. Mystical Bedlam: Madness, Anxiety and Healing in Seventeenth Century England. Cambridge: Cambridge University Press.

Machiavelli, Niccolò. 1962 [1532]. Il Principe e altri scritti. Vittorio De Caprariis (a cura di). Bari: Laterza.

Machiavelli, Niccolò. 2000 [1531]. Discorsi sopra la prima Deca di Tito Livio. Corrado Vivanti (a cura di). Torino: Einaudi.

Natali, Ilaria. 2016. "Remov'd from human eyes": Madness and Poetry 1676-1774. Firenze: Firenze University Press. 
Nicoll, Allardyce. 1952 [1923]. A History of Restoration Drama 1600-1700. Cambridge: Cambridge University Press.

Porter, Roy. 2000. Enlightenment: Britain and the Creation of the Modern World. London: The Penguin Press.

Porter, Roy. 2002. Madness: A Brief History. Oxford: Oxford University Press.

Scull, Andrew T. 2015. Madness and Civilization. A Cultural History of Insanity from the Bible to Freud, from the Madhouse to Modern Medicine. Princeton: Princeton University Press.

Verdurmen, Peter. 1989. Lucius Junius Brutus and Restoration Tragedy: The Politics of Trauma. Journal of European Studies, 19, 2: 81-98.

Vieth, David M. 1975. Psychological Myth as Tragedy: Nathaniel Lee's Lucius Junius Brutus. Huntington Library Quarterly, 39, 1: 57-76.

Ward, William Adolphus. 1875. A History of English Dramatic Literature to the Death of Queen Anne (Vol. 2). London: Macmillan \& Co.

Willis, Thomas. 1683. Two Discourses Concerning the Soul of Brutes. London: Thomas Dring. Willis, Thomas. 1685 [1681]. The London Practice of Physick. London: Thomas Basset et al. Wilmot John, Earl of Rochester. 1800 [1675]. An Allusion to the Tenth Satire of the First Book of Horace. Samuel Johnson ed. The Works of the Poets of Great Britain and Ireland (Vol. II). London: Andrew Miller, 16-17.

Wilson, John Harold. 1968. A Preface to Restoration Drama. Cambridge (MA): Harvard University Press.

Ilaria Natali insegna Letteratura inglese presso l'Università di Firenze. Si occupa principalmente di analisi di manoscritti moderni, con particolare attenzione all'opera di James Joyce e altri autori del Modernismo. Ha approfondito vari aspetti della letteratura e della cultura irlandese dell'Ottocento e del Novecento in articoli e curatele. Dal 2016 ha maturato un interesse per le relazioni tra letteratura e storia della medicina, dedicando vari studi alla follia nella poesia inglese del Settecento. Il suo quinto libro, Reading the Inferno: James Joyce's Notazioni on Dante's Divine Comedy, è pubblicato da Bulzoni nel 2020. ilaria.natali@unifi.it 